Document downloaded from:

http://hdl.handle.net/10251/108048

This paper must be cited as:

Merli Gisbert, R.; Monleón Cremades, S.; Lazaro, C. (2017). Energy approach to the unstressed geometry of single-walled carbon nanotubes. Meccanica. 52(1-2):213-230. doi:10.1007/s11012-016-0389-z

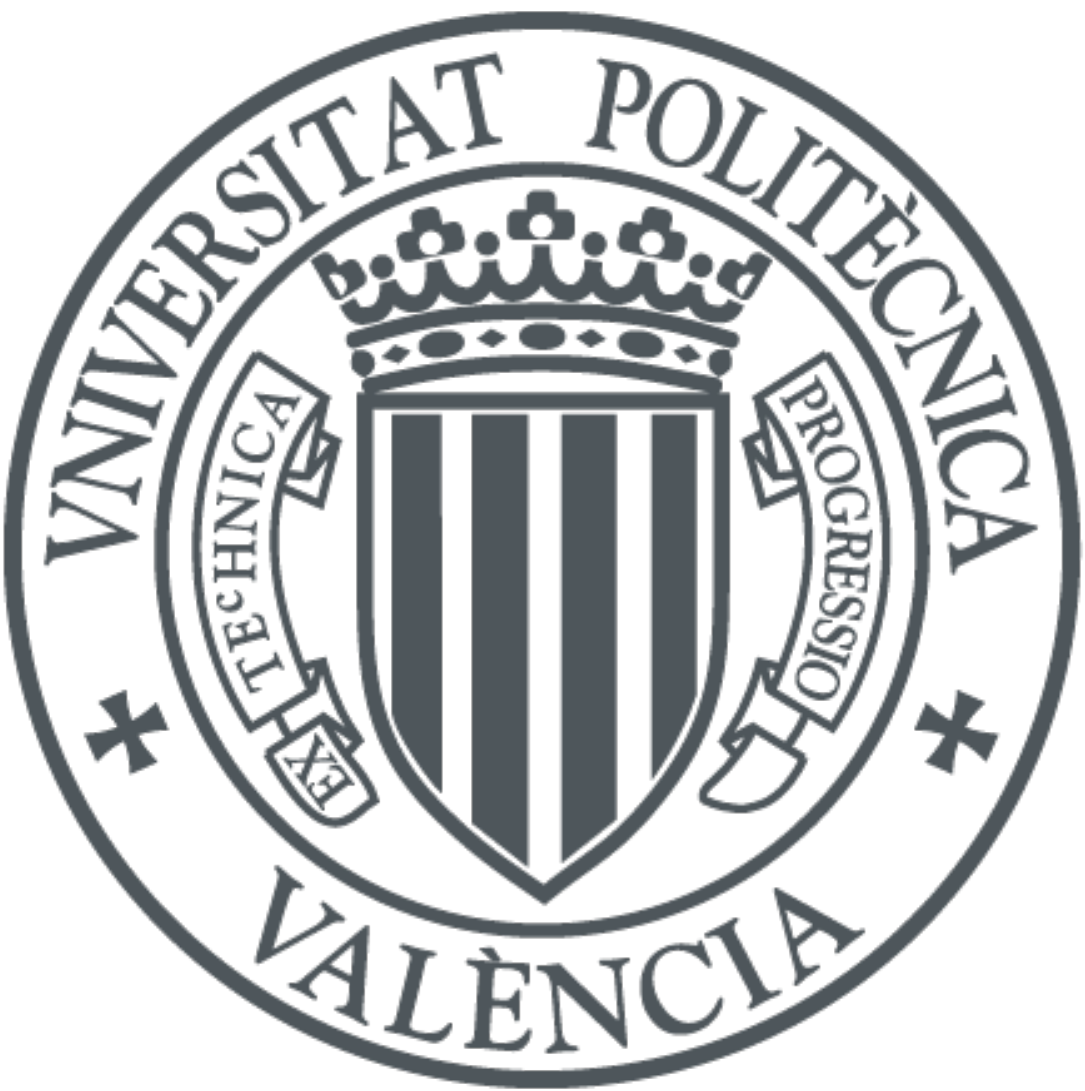

The final publication is available at

https://doi.org/10.1007/s11012-016-0389-z

Copyright Springer-Verlag

Additional Information 


\title{
Energy approach to the unstressed geometry of single-walled carbon nanotubes
}

\author{
Rafael Merli · Salvador Monleón · Carlos Lázaro
}

the date of receipt and acceptance should be inserted later

\begin{abstract}
In this paper, the geometry of single-walled carbon nanotubes without any external loading is analyzed via an energy procedure. The nanotube is assumed to be inscribed into a perfect cylinder of unknown diameter, which is estimated by minimizing the total interatomic potential involved into a basic cell with several carbon atoms and their corresponding bonds. In this step, two interatomic potentials have been adopted in order to compare their influence on the obtained results. Our calculations show that the widely used conformal mapping is not the most suitable option to reproduce the geometry of single-walled nanotubes in absence of external loading. Likewise, a more accurate method to estimate the initial diameter of the nanotube is developed, yielding higher differences with smaller nanotubes in comparison with other published works .

The present analysis can be useful in the framework of Molecular Mechanics or continuum models as an alternative way to introduce initial stresses (due to the curvature of the cylinder) in the mechanical analysis, against other involved methods.
\end{abstract}

Keywords Carbon nanotubes - Molecular mechanics · Energy minimization $\cdot$ Prestressed state

\section{Introduction}

Carbon nanotubes (CNTs) have been a remarkable centre of attention into the scientific and research community over the past two decades, due to their outstanding mechanical and

R. Merli (corresponding author) · C. Lázaro $\cdot \mathrm{S}$. Monleón

Departamento de Mecánica de los Medios Continuos y Teoría de Estructuras. Escuela Técnica Superior de Ingenieros de Caminos, Canales y Puertos. Universitat Politècnica de València. Camino de vera s/n, 46022 Valencia, Spain

Tel:+3496387 76 77, Fax: +34963879679

E-mail: ramergis@doctor.upv.es electrical properties [7]. For instance, CNTs show a singular coupling between mechanical strain and electrical conductivity $\{21,22 \mid$, becoming ideal candidates for making nanosensors and nano electro-mechanical systems (NEMS), with promising applications in robotics and biomechanics.

Regarding Materials Science and Engineering, nanotube reinforced composites and polymers have shown a wide field of potential applications, specially where a high strengthto-weight ratio is needed (e.g. aircraft industry). The main structural properties are their extreme longitudinal stiffness $[8]$ (Young's modulus $\simeq 1 \mathrm{TPa}$ ) and tensile strength $|3|$ $\left(\sigma_{y} \simeq 50 \mathrm{GPa}\right)$.

The present work is focused on single-walled carbon nanotubes (SWCNTs), which may be conceptualized as the result of rolling up a graphene sheet into a cylinder. Into each graphene sheet, Carbon atoms are arranged in a covalentbonded honeycomb lattice.

Prior to the practical application of nanotubes in manufacturing composites (as well as other structural applications), a high understanding of their mechanical behavior is needed. However, to date, there is no experimental work about individual SWCNTs because their extremely small size makes difficult to handle these nanomolecules. Therefore, theoretical work is required in order to analyze the mechanical response of SWCNTs.

The existing analytical or numerical methods applied to the mechanical behavior of nanotubes, can be roughly classified in two main categories: atomistic scale and continuum scale methods. The atomistic methods, for instance Molecular Dynamics (MD), ab initio or tight-binding, can successfully reproduce physical phenomena as buckling [12 29| and estimate elastic parameters of CNTs [21], but they have the disadvantage of being limited at a relatively low number of atoms (about $10^{9}$ according to [27|) because of their high computational cost. 
Otherwise, continuum methods are capable of analyze longer systems, but the equivalence with the atomistic level is not clear. In fact, the wall thickness adopted to reproduce the nanotube mechanical response through an equivalent beam or shell model ranges from $t=0.066 \mathrm{~nm} \mathrm{[29]}$ to

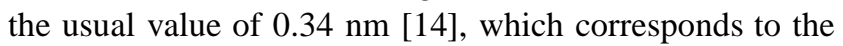
inter-planar distance in graphite. Even some authors deem CNTs as solid cylinders [27|. In addition, continuum methods can not reflect the atomistic detail which may have an important influence on the final response of CNTs.

As a compromise between both groups of models, Molecular Structural Mechanics (MSM) models are reasonable in terms of computational expense whereas atomistic scale is correctly displayed. Some previous works $|10,11,30|$ considered CNTs as a frame system with carbon atoms located at nodes and rigid bars (provided with axial, flexural and torsional stiffnesses) representing covalent bonds. Different layers in multi-walled carbon nanotubes (MWCNTs) were connected by several truss rods between neighboring atoms. Alternatively, [19] modeled the graphene sheet as a 2D truss model with additional rods through the unit hexagonal cell.

Included in the MSM models, the 'stick-spiral' model introduced by $[5]$ reproduces covalent bonds by axial springs and the three-body interaction by three spiral springs on each node. Since that work, several studies $[17,18,26,28]$ tackled the determination of mechanical parameters of SWCNTs through the 'stick-spiral' model, but taking advantage of the axisymmetry of ZigZag and Armchair nanotubes and limiting their analysis to a small unit cell involving only a few atoms. Although $|6|$ generalized the work in $[5]$ to Chiral SWCNTs, the study is also restricted to representative cell which reproduces the geometry of Chiral nanotubes by means of their helicoidal symmetry.

From a more general point of view, [14] investigated the longitudinal behavior of SWCNTs (even with Chiral nanotubes) by implementing the 'stick-spiral' model in the commercial code ANSYS ${ }^{\circledR}$, but including the whole geometry of the nanotube in their simulations. In this line, [15] developed a general formulation for the same model able to reproduce any loading and supporting distribution, oriented to obtain the mechanical parameters of SWCNTs. Furthermore, in $[16]$ the same authors extended the formulation to calculate buckling strains under several loading schemes.

A specially important issue, mainly in atomistic models is the preenergy, defined as the excess of strain energy from an infinite planar graphene sheet to the nanotube $[9,29]$. Associated with this variation of strain energy, a system of internal stresses and strains in the nanotube will appear, leading to a stabilization effect into its cross-sectional area. In fact, $|25|$ studied the influence of the radius on the transversal deformation due to the Van der Waals (VDW) interaction in a set of SWCNTs and concluded that the flattening of the cross-section increased with the radius. However, for nan- otubes smaller in diameter $(R<10 \AA)$, where the preenergy is determining compared with the VDW interaction, SWCNTs kept their cylindrical geometry. Likewise, [8, 20, 23] have shown that the preenergy is proportional to the curvature of the wall $1 / R^{2}$ (where $\mathrm{R}$ is the tube radius).

Since the honeycomb lattice of Carbon atoms in the nanotube has a complex behavior, it is inaccurate to state that the initial equilibrium configuration of SWCNTs is a perfect cylinder. In fact, some authors $[4,13]$ have proved by means of nonlocal continuum models, that SWCNTs subjected to tensile stresses or electromechanically actuated, present end effects which separates the nanotube geometry from the cylindrical shape. Within atomistic simulations, the approach to the equilibrium geometry is often introduced $[1,9,12,24,29]$ by decomposing the loading process into multiple stages and minimizing the total energy into each stage by means of some numerical algorithm (e.g. dynamic relaxation). Nevertheless, taking into account MSM models are rather deterministic regarding the geometry, in $\mid 15]$ the preenergy is introduced as a system of initial strains which produces a 'prestressed state' previous to the action of external loads. As the structural system is statically undetermined, there are many possible sets of initial strains and stresses in equilibrium without external loads. To overcome this hurdle, the usually proposed conformal mapping (see e.g. $[5,7,14,28 \mid)$ was adopted in such a way that Carbon atoms are kept on the cylindrical surface involving the nanotube, while covalent bonds are located along secants among two covalent-bonded atoms. In this sense, the estimation of initial stresses is closely related to the choice of the specific mapping adopted to reproduce the geometry of SWCNTs.

The main reason to use the conformal mapping from the planar graphene sheet to a cylinder is the simplicity of its analytical formulation. Therefore, some accuracy is missing in order to approach the problem in a straightforward manner. Nonetheless, in this paper a better approach to the initial geometry of the nanotube is studied by means of an energy minimization procedure. It is expected that initial stresses in this improved geometry will be lower in comparison with those obtained from the conformal mapping. In particular, if stresses are small enough, the formulation of the "stickspiral' model in $[15 \mid$ may be simplified by neglecting the terms corresponding to the preenergy leading to a simpler numerical implementation. In addition, a more accurate obtention of the initial geometry of SWCNTs hopefully provides more reliable results of stresses, strains and buckling patterns once external loads are applied.

In this work, the nanotube is assumed to be inscribed into a perfect cylinder. Thus, our modeling of the geometry does not incorporate the aforementioned end effects, since they are considered to be locally concentrated in small regions around both ends in long nanotubes. The obtention of the initial geometry may be tackled in two steps. First, 
the diameter of the cylinder should be obtained, which is worked out in the present paper. Secondly, a new analytical mapping is required to determine the location of Carbon atoms on the cylinder, which will be treated in further research.

In this paper, the main objective is calculating the diameter for the unstressed geometry (assuming it is a perfect cylinder) of ZigZag, Armchair and Chiral SWCNTs by using a energy approach, and comparing results between AM$\mathrm{BER}^{1}$ and Morse interatomic potentials (a detailed description is given in [15|). A method has been developed for this purpose, in which analytical expressions have been derived and numerically implemented. The present work improves the theoretical approach to the initial geometry of the nanotube provided by the widely used conformal mapping.

As has been mentioned before, if the conformal mapping is strictly employed, a set of initial forces between atoms (and initial moments among neighboring bonds) should be considered due to this mapping modifies the interatomic distances (and angles between bonds) with respect to their corresponding values in the planar graphene sheet. Such effect has been evaluated in $|15|$. This study may be regarded as a first step oriented to establish an alternative to the direct estimation of initial strains and stresses, which hopefully may simplify the numerical formulation of the 'stick-spiral' model.

The comparison of the obtained diameters with those provided by the conformal mapping allows to study the accuracy of the latter against the size of the nanotube.

The paper is organized as follows: in section 2 , the main assumptions and a brief description of the interatomic potentials are provided. From section 3 to section 5, the main equations and results of the energy approach are developed for ZigZag, Armchair and Chiral nanotubes, respectively. Finally, concluding remarks are addressed in section 6 . As additional content, in appendix $\mathrm{A}$ the validation of the approximate mapping adopted in Chiral nanotubes is included. In appendix B estimation of error between AMBER and Morse potentials into the energy approach is justified.

\section{Initial assumptions and interatomic potentials}

In order to find the diameter of unstressed nanotubes, the following assumptions have been made:

1. The initial geometry of the nanotube is such that the atoms are contained into a perfect infinite cylinder of diameter $d_{0}$.

2. There is no external load acting on the nanotube.

\footnotetext{
1 Assisted Model Building with Energy Refinement, force field well-known in biomolecular simulation.
}

3. The total energy of the system is defined by the interatomic potential under small strains assumption. Therefore, terms related to electrostatic, VDW, torsion and inversion interactions are neglected.

4. Since the longitudinal stiffness of covalent bonds is significantly higher than angular stiffness between two neighboring covalent bonds, the lengthening of covalent bonds from the planar graphene sheet to the nanotube has been neglected. Therefore, in the equilibrium state all bonds are $a_{0}=0.142 \mathrm{~nm}$ in length |7|.

The basic procedure to calculate initial diameters will be to minimize the total energy of the system, taking into account two different interatomic potentials. Regarding assumption 3, the AMBER potential (see e.g. |5|, Eq. 8) can be expressed as:

$U=\sum U_{r}+\sum U_{\theta}=\sum_{i} \frac{1}{2} k_{r}\left(\Delta r_{i}\right)^{2}+\sum_{j} \frac{1}{2} k_{\theta}\left(\Delta \theta_{j}\right)^{2}$,

where

$\Delta r_{i} \quad$ lengthening of the bond $\mathrm{i}$

$\Delta \theta_{j} \quad$ change in angle between two neighboring covalent bonds involving the angle $\mathrm{j}$

$k_{r}, k_{\theta} \quad$ force constants to the longitudinal and angular variations respectively

Following [10 171926 30], the next values are adopted:

$$
k_{r}=652 \frac{\mathrm{nN}}{\mathrm{nm}}, \quad k_{\theta}=0.876 \frac{\mathrm{nN} \cdot \mathrm{nm}}{\mathrm{rad}} .
$$

Taking into account assumption 4, Eq. (1) can be simplified as:

$U=\sum_{j} \frac{1}{2} k_{\theta}\left(\Delta \theta_{j}\right)^{2}$.

Hence, the force constant $k_{r}$ is not involved in our calculations. However, its value is included in (5) to complete definition (1).

On the other hand, the Morse potential can be written as:

$$
\begin{aligned}
U=\sum_{i} D_{e}\left\{\left[1-e^{-\beta\left(\Delta r_{i}\right)}\right]^{2}-1\right\}+ \\
+\sum_{j} \frac{1}{2} k_{\theta}\left(\Delta \theta_{j}\right)^{2}\left[1+k_{s}\left(\Delta \theta_{j}\right)^{4}\right],
\end{aligned}
$$

where the parameters involved have been taken from table 1 in [3], namely:

$$
\begin{array}{rlrl}
D_{e} & =0.2895 \mathrm{nN} \cdot \mathrm{nm}, & & \beta=38.43 \mathrm{~nm}^{-1}, \\
k_{\theta}=0.8998 \mathrm{nN} \cdot \mathrm{nm}, & k_{s}=0.754 \mathrm{rad}^{-4},
\end{array}
$$

and regarding assumption 4, Eq. (4) can be simplified as:

$$
U=-\sum_{i} D_{e}+\sum_{j} \frac{1}{2} k_{\theta}\left(\Delta \theta_{j}\right)^{2}\left[1+k_{s}\left(\Delta \theta_{j}\right)^{4}\right] .
$$


As the angular distortion $\Delta \theta_{j}$ can be expressed as a function of the nanotube diameter $d$, Eqs (3) and (6) adopt the form $U=U(d)$. Therefore, the diameter $d_{0}$ corresponding to the initial equilibrium geometry should verify the total energy minimum condition:

$\left[\frac{\partial U(d)}{\partial d}\right]_{d_{0}}=0$.

In the following sections, Eq. (7) is developed and solved for each chirality.

\section{Initial diameter for ZigZag nanotubes}

Zig-zag nanotubes (denoted herein as $\mathrm{ZZ}(\mathrm{m}, 0)$ ) are composed by $m$ cells in the transversal direction and possess axial symmetry. An infinite SWCNT of this type can be generated by repeating in the axial direction the elemental system depicted in figure 1 . Thus, the energy minimization (7) can be carried out over such system without loss of generality.

Since all bond lengths are $a_{0}$ in the equilibrium position (no lengthening), we can write:

$l=a_{0} \cos \alpha$

$\frac{l}{2}=R \sin \left(\frac{\pi}{2 m}\right)$,

where:

$l$ projection of the bond length on the cross section of the nanotube

$\alpha$ angle included from each oblique bond and the transversal plane

Removing $l$ from eq. (8), the next relation is reached:

$d_{0}=2 R=\frac{a_{0} \cos \alpha}{\sin \left(\frac{\pi}{2 m}\right)}$.

For the sake of simplicity, we adopt $\alpha$ as independent parameter in the minimization process.

From figure 1, can be observed that there are only two different sets of bond angles into our elemental system. The first of them, included between bonds a-c and b-c, has a value of:

$\theta_{1}=\alpha+\frac{\pi}{2}$

The second angle $\theta_{2}$ is the one formed by two consecutive bonds a-b. The next diagrams (including three neighboring atoms) depicted in figure 2 show this angle.

As can be seen, the coordinate system adopted in figure 2(b) includes the bond A-B in its $\{y z\}$-plane. Thus, the following auxiliary vectors may be introduced:

$\mathbf{v}_{1}=\overrightarrow{A B}=\left\{0,-a_{0} \cos \alpha, a_{0} \sin \alpha\right\}^{T}$,

$\mathbf{v}_{2}=\overrightarrow{A B^{\prime}}=\left\{-a_{0} \cos \alpha \sin \left(\frac{\pi}{m}\right), a_{0} \cos \alpha \cos \left(\frac{\pi}{m}\right), a_{0} \sin \alpha\right\}^{T}$,
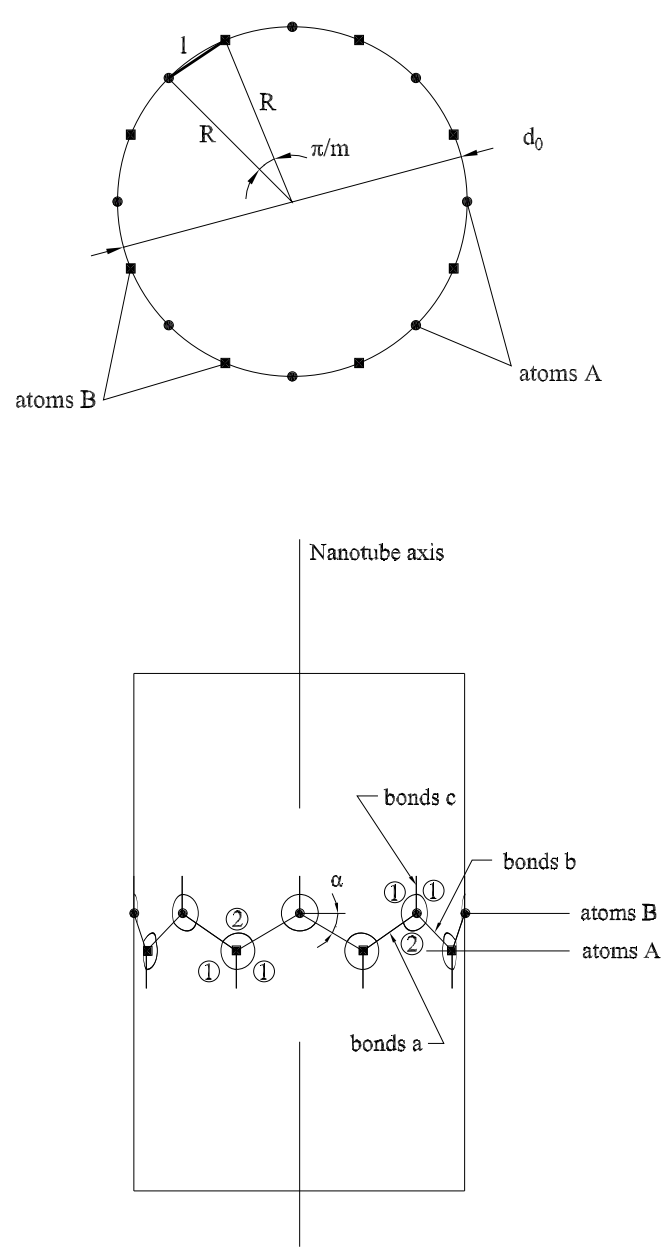

Fig. 1 Representative cell for Zig-zag nanotubes

obtaining the angle $\theta_{2}$ from their dot product:

$\cos \theta_{2}=\sin ^{2} \alpha\left(1+\cos \frac{\pi}{m}\right)-\cos \frac{\pi}{m}$.

Since in the planar graphene sheet all angles between bonds are equal to $\frac{2 \pi}{3}$, from equations (10) and (12), the angular distortions from the plane configuration can be expressed as:

$\Delta \theta_{1}=\alpha-\frac{\pi}{6}$,

$\Delta \theta_{2}=\arccos \left[\sin ^{2} \alpha\left(1+C_{m}\right)-C_{m}\right]-\frac{2 \pi}{3}$,

where:

$C_{m}=\cos \left(\frac{\pi}{m}\right)$.

\subsection{Energy minimization with AMBER potential}

Applying the AMBER potential function (3) to our elementary system (see figure 1), which includes $4 m$ angles of type 


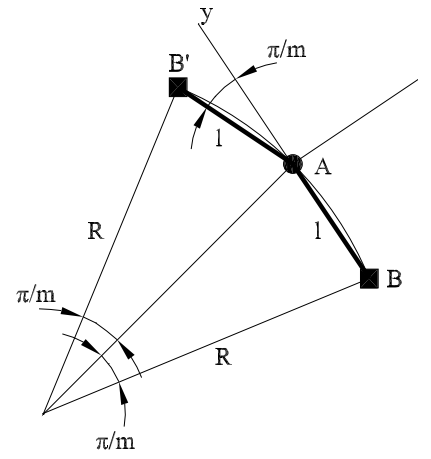

(a) Cross section

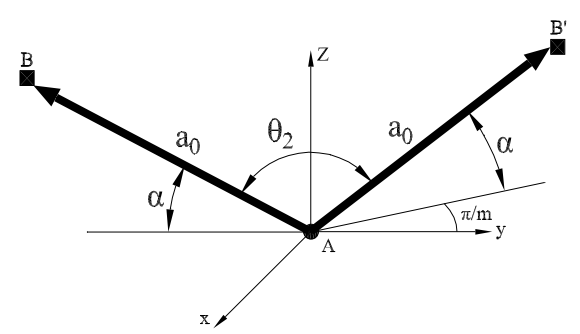

(b) Perspective

Fig. 2 Diagrams to obtain $\theta_{2}$

$\theta_{1}$ and $2 m$ angles of type $\theta_{2}$, the next expression is reached:

$U=m k_{\theta}\left(\Delta \theta_{2}\right)^{2}+2 m k_{\theta}\left(\Delta \theta_{1}\right)^{2}$.

Forcing the minimum condition (7) into (15)

$m k_{\theta}\left(2 \Delta \theta_{2} \frac{\partial \Delta \theta_{2}}{\partial \alpha}+4 \Delta \theta_{1} \frac{\partial \Delta \theta_{1}}{\partial \alpha}\right)=0$,

introducing (13) in (16) and operating, leads to:

$$
\begin{aligned}
-\left\{\arccos \left[\sin ^{2} \alpha\left(1+C_{m}\right)-C_{m}\right]-\frac{2 \pi}{3}\right\} & \\
& \cdot \frac{\left(1+C_{m}\right) \sin \alpha \cos \alpha}{\sqrt{1-\left[\sin ^{2} \alpha\left(1+C_{m}\right)-C_{m}\right]^{2}}}+\left(\alpha-\frac{\pi}{6}\right)=0 .
\end{aligned}
$$

The latter expression is a nonlinear equation of $\alpha$, which was solved numerically by the Newton method. Once $\alpha$ is obtained and substituting in (9), the values of diameter $d_{0}$ outlined in table 1 are obtained for $a_{0}=0.142 \mathrm{~nm}$. Likewise, the diameters $D_{t}$ calculated with the conformal mapping [7] and the relative error taking $d_{0}$ as the reference solution are included to get an insight into the difference.

As has been shown in table 1 , our obtained diameters $d_{0}$ are slightly higher than those obtained from the conformal mapping. This difference is probably related to the stabilization effect of the preenergy, which tend to expand transversally the nanotube. The smaller the diameter is, the higher

\begin{tabular}{lccccc}
\hline & $\mathrm{m}$ & $\alpha(\mathrm{rad})$ & $d_{0}(\mathrm{~nm})$ & $D_{t}(\mathrm{~nm})$ & $\varepsilon(\%)$ \\
\hline $\mathrm{ZZ}(4,0)$ & 4 & 0.4406 & 0.3356 & 0.3132 & 6.6746 \\
$\mathrm{ZZ}(5,0)$ & 5 & 0.4685 & 0.4100 & 0.3914 & 4.5366 \\
$\mathrm{ZZ}(6,0)$ & 6 & 0.4848 & 0.4854 & 0.4697 & 3.2344 \\
$\mathrm{ZZ}(10,0)$ & 10 & 0.5094 & 0.7925 & 0.7829 & 1.2114 \\
$\mathrm{ZZ}(15,0)$ & 15 & 0.5173 & 1.1808 & 1.1743 & 0.5505 \\
$\mathrm{ZZ}(20,0)$ & 20 & 0.5200 & 1.5706 & 1.5658 & 0.3056 \\
\hline
\end{tabular}

Table 1 Initial diameters ZigZag, AMBER. $D_{t}$ is the diameter associated to the conformal mapping, $d_{0}$ is the obtained diameter from the minimization procedure and $\varepsilon(\%)$ represents the relative error taking $d_{0}$ as the reference solution

effect of the preenergy is found. Following $|8,20,23|$, a curve of the form $\varepsilon=k / R^{2}$ can be fitted by imposing it passes through the first point ( $k=0.1879$ is obtained). Plotting the obtained relative error and the fitted curve against the diameter in figure 3 , almost full agreement is found.

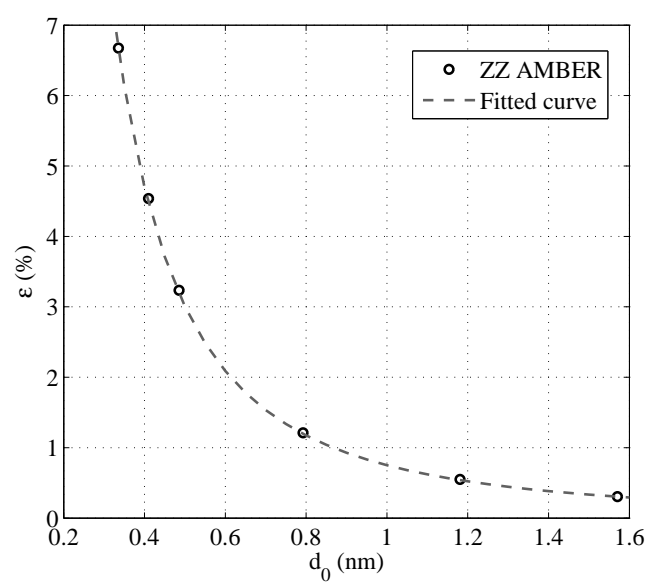

Fig. 3 Relative error against diameter (ZigZag, AMBER)

From figure 3 , it may be remarked that a set of initial forces and moments should be explicitly included (mainly in MSM models) for smaller diameters when the conformal mapping is adopted, specially for diameters below $0.8 \mathrm{~nm}$ $(\varepsilon>1 \%)$.

\subsection{Energy minimization with Morse potential}

The expression (6) of Morse potential reduced to the elementary system in figure 1 can be written:

$$
\begin{aligned}
U=-4 m D_{e}+m k_{\theta}\left(\Delta \theta_{2}\right)^{2}\left[1+k_{s}\left(\Delta \theta_{2}\right)^{4}\right]+ \\
+2 m k_{\theta}\left(\Delta \theta_{1}\right)^{2}\left[1+k_{s}\left(\Delta \theta_{1}\right)^{4}\right] .
\end{aligned}
$$


Forcing the minimum condition ( 7 ) into (18), the next equation is reached:

$$
\begin{aligned}
-\Delta \theta_{2}\left[1+3 k_{s}\left(\Delta \theta_{2}\right)^{4}\right] & \frac{\left(1+C_{m}\right) \sin \alpha \cos \alpha}{\sqrt{1-\left[\sin ^{2} \alpha\left(1+C_{m}\right)-C_{m}\right]^{2}}}+ \\
& +\Delta \theta_{1}\left[1+3 k_{s}\left(\Delta \theta_{1}\right)^{4}\right]=0,
\end{aligned}
$$

where $\Delta \theta_{1}, \Delta \theta_{2}$ are functions of $\alpha$ through (13). Thus, (19) is an implicit equation of $\alpha$. Solving it numerically by the Newton method for $a_{0}=0.142 \mathrm{~nm}$ and substituting in (9), the initial diameter without external loading is rendered. The obtained values of $d_{0}$ (not reproduced herein) were the same as those in table 1 , because the absolute error in the minimum condition (7) between AMBER and Morse potentials is lower than the accuracy $\left( \pm 10^{-4}\right)$ adopted for $d_{0}$ (see appendix $B$, table 6). Hence, it can be concluded that the initial diameter of ZigZag nanotubes is not influenced by the choice of interatomic potential.

\section{Initial diameters for Armchair nanotubes}

The elemental system (including $2 m$ cells in the transversal direction) which generates an Armchair nanotube (named herein as $\mathrm{AC}(\mathrm{m}, \mathrm{m})$ ) by repetition in the axial direction, is represented in figure 4 . In this section, the initial equilibrium geometry of an Armchair SWCNT is studied by minimizing the total potential energy of such a system.

From figure 4 and the diagram in figure 5(a), the next relations may be established:

$l=a_{0} \sin \alpha$,

$\frac{a_{0}}{d_{0}}=\sin \frac{\theta}{2}, \quad \sqrt{1-\left(\frac{a_{0}}{d_{0}}\right)^{2}}=\cos \frac{\theta}{2}$,

$\frac{l}{d_{0}}=\sin \frac{\varphi}{2}, \quad \sqrt{1-\left(\frac{l}{d_{0}}\right)^{2}}=\cos \frac{\varphi}{2}$.

In order to relate the nanotube diameter $d_{0}$ to the angle $\alpha$ (see fig. 5(b)), we can write:

$\sin \left(\frac{\theta}{2}+\frac{\varphi}{2}\right)=\sin \frac{\theta}{2} \cos \frac{\varphi}{2}+\sin \frac{\varphi}{2} \cos \frac{\theta}{2}=\sin \frac{\pi}{2 m}$,

$\cos \left(\frac{\theta}{2}+\frac{\varphi}{2}\right)=\cos \frac{\theta}{2} \cos \frac{\varphi}{2}-\sin \frac{\varphi}{2} \sin \frac{\theta}{2}=\cos \frac{\pi}{2 m}$.

Squaring (21) and substituting (22), next expression is reached:

$$
\begin{aligned}
\sin ^{2} \frac{\theta}{2} \cos ^{2} \frac{\varphi}{2}+\cos ^{2} \frac{\theta}{2} \sin ^{2} \frac{\varphi}{2}+2 \sin ^{2} \frac{\theta}{2} \sin ^{2} \frac{\varphi}{2}+ \\
+2 \sin \frac{\theta}{2} \sin \frac{\varphi}{2} \cos \frac{\pi}{2 m}=\sin ^{2} \frac{\pi}{2 m}
\end{aligned}
$$
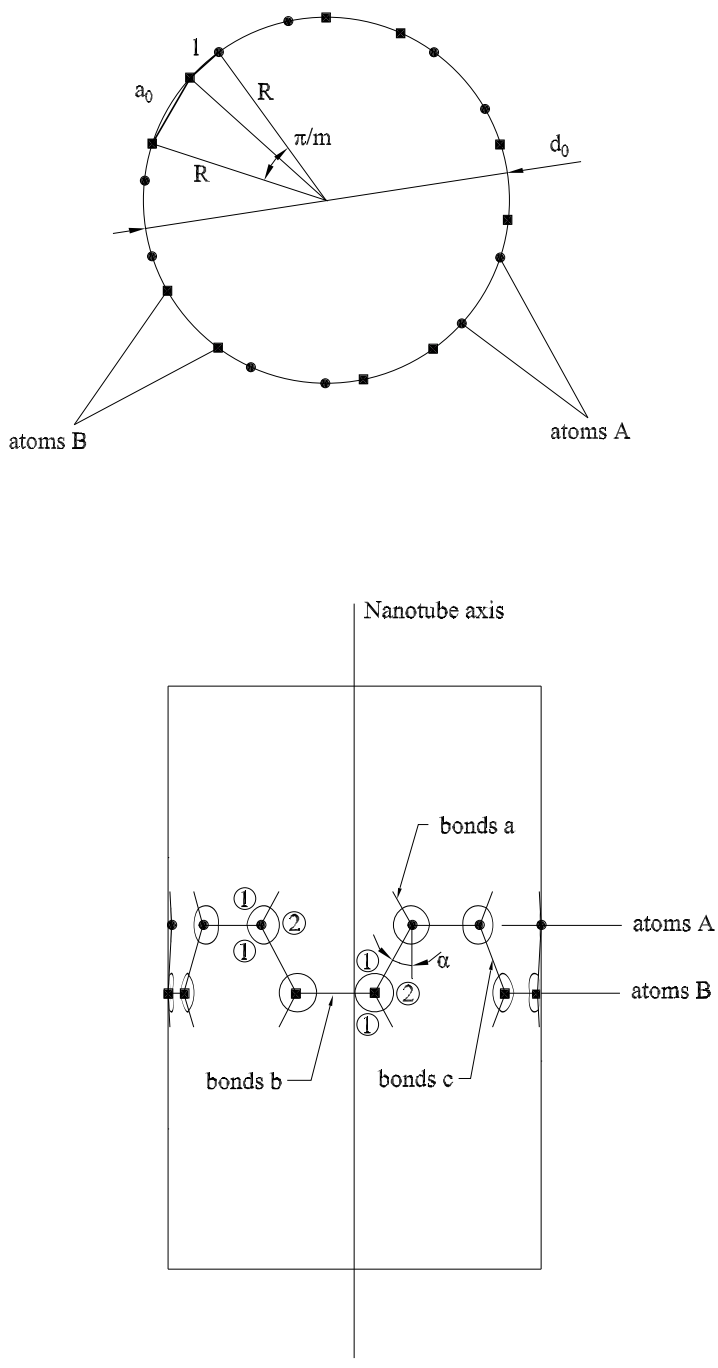

Fig. 4 Representative cells for Armchair nanotubes

Substituting (20) into (23) and operating

$d_{0}=\frac{a_{0}}{S_{2 m}} \sqrt{1+\sin ^{2} \alpha+2 \sin \alpha C_{2 m}}$,

where:

$S_{2 m}=\sin \frac{\pi}{2 m}, \quad C_{2 m}=\cos \frac{\pi}{2 m}$.

Similarly to the case of $\mathrm{ZZ}$ nanotubes, the angle $\alpha$ is adopted as independent parameter in the minimization process for Armchair SWCNTs. Therefore, relations of the angular distortions $\Delta \theta_{1}, \Delta \theta_{2}$ as functions of $\alpha$ are needed. For this purpose, figure 5(b) is useful to define the next auxiliary vec- 


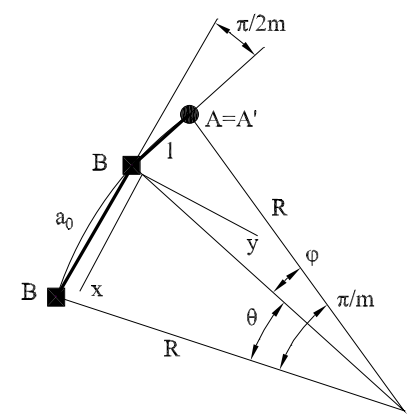

(a) Cross section

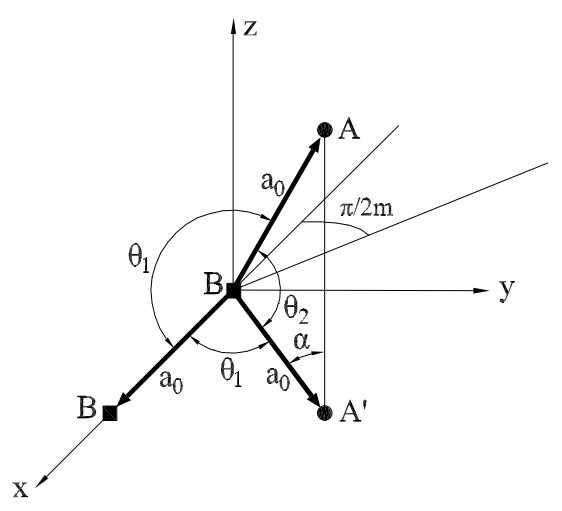

(b) Perspective

Fig. 5 Diagrams to obtain $\theta_{1}, \theta_{2}$

tors:

$\mathbf{w}_{1}=\overrightarrow{B B}=\left\{a_{0}, 0,0\right\}^{T}$,

$\mathbf{w}_{2}=\overrightarrow{B A}=\left\{-a_{0} \sin \alpha C_{2 m}, a_{0} \sin \alpha S_{2 m}, a_{0} \cos \alpha\right\}^{T}$,

$\mathbf{w}_{3}=\overrightarrow{B A^{\prime}}=\left\{-a_{0} \sin \alpha C_{2 m}, a_{0} \sin \alpha S_{2 m},-a_{0} \cos \alpha\right\}^{T}$.

The dot products $\mathbf{w}_{1}^{T} \mathbf{w}_{2}$ and $\mathbf{w}_{2}^{T} \mathbf{w}_{3}$ allow to calculate, respectively

$\cos \theta_{1}=-C_{2 m} \sin \alpha$,

$\cos \theta_{2}=-\cos 2 \alpha$.

From (27b), it follows $\theta_{2}=\pi-2 \alpha$. Thereby, the required angular distortions for $\mathrm{AC}$ nanotubes are written:

$\Delta \theta_{1}=\arccos \left[-C_{2 m} \sin \alpha\right]-\frac{2 \pi}{3}$,

$\Delta \theta_{2}=\frac{\pi}{3}-2 \alpha$.

\subsection{Energy minimization with AMBER potential}

As can be deduced from figure 4 , the elemental system representing an Armchair nanotube includes $4 m$ angles referred to as 2 and $8 m$ angles noted as 2 . Thus, the expression (3) for AMBER potential is converted into:

$U=2 m k_{\theta}\left(\Delta \theta_{2}\right)^{2}+4 m k_{\theta}\left(\Delta \theta_{1}\right)^{2}=2 m k_{\theta}\left(\Delta \theta_{2}^{2}+2 \Delta \theta_{1}^{2}\right)$.

Applying the minimum condition (7) to (29) and introducing the angular distortions (28), the next implicit equation of $\alpha$ is achieved:

$$
\begin{array}{r}
\left(\arccos \left[-C_{2 m} \sin \alpha\right]-\frac{2 \pi}{3}\right) \frac{C_{2 m} \cos \alpha}{\sqrt{1-\left(C_{2 m} \sin \alpha\right)^{2}}}- \\
-\left(\frac{\pi}{3}-2 \alpha\right)=0
\end{array}
$$

The equation (30) is solved by using the Newton method and the optimized diameter $d_{0}$ computed by means of (24) for $a_{0}=0.142 \mathrm{~nm}$. An outline of the obtained values in comparison with the corresponding to the conformal mapping $D_{t}$ is included in table 2 . As can be expected, our values of $d_{0}$ for AC nanotubes are slightly higher than those calculated from the conformal mapping.

\begin{tabular}{lccccc}
\hline & $\mathrm{m}$ & $\alpha(\mathrm{rad})$ & $d_{0}(\mathrm{~nm})$ & $D_{t}(\mathrm{~nm})$ & $\varepsilon(\%)$ \\
\hline $\mathrm{AC}(3,3)$ & 3 & 0.5470 & 0.4185 & 0.4068 & 2.7957 \\
$\mathrm{AC}(4,4)$ & 4 & 0.5375 & 0.5514 & 0.5424 & 1.6322 \\
$\mathrm{AC}(5,5)$ & 5 & 0.5327 & 0.6853 & 0.6780 & 1.0652 \\
$\mathrm{AC}(10,10)$ & 10 & 0.5260 & 1.3597 & 1.3560 & 0.2721 \\
$\mathrm{AC}(15,15)$ & 15 & 0.5246 & 2.0365 & 2.0340 & 0.1228 \\
$\mathrm{AC}(20,20)$ & 20 & 0.5242 & 2.7139 & 2.7120 & 0.0700 \\
\hline
\end{tabular}

Table 2 Initial diameters Armchair, AMBER. $D_{t}$ is the diameter associated to the conformal mapping, $d_{0}$ is the obtained diameter from the minimization procedure and $\varepsilon(\%)$ represents the relative error taking $d_{0}$ as the reference solution

Taking $d_{0}$ as the reference solution, relative errors in the last column of table 2 have been obtained. As has been done for ZigZag nanotubes, a curve proportional to $1 / R^{2}$ can be fitted [ 82023 ] to the relative errors for Armchair nanotubes, rendering $\varepsilon=0.2091 / R^{2}$. Both curves are quite similar, as can be shown in figure 6

\subsection{Energy minimization with Morse potential}

The expression (6) of Morse potential reduced to the elemental system of figure 4 takes a form:

$$
\begin{aligned}
U=-12 m D_{e}+2 m k_{\theta}\left(\Delta \theta_{2}\right)^{2}\left[1+k_{s}\left(\Delta \theta_{2}\right)^{4}\right]+ \\
+4 m k_{\theta}\left(\Delta \theta_{1}\right)^{2}\left[1+k_{s}\left(\Delta \theta_{1}\right)^{4}\right] .
\end{aligned}
$$




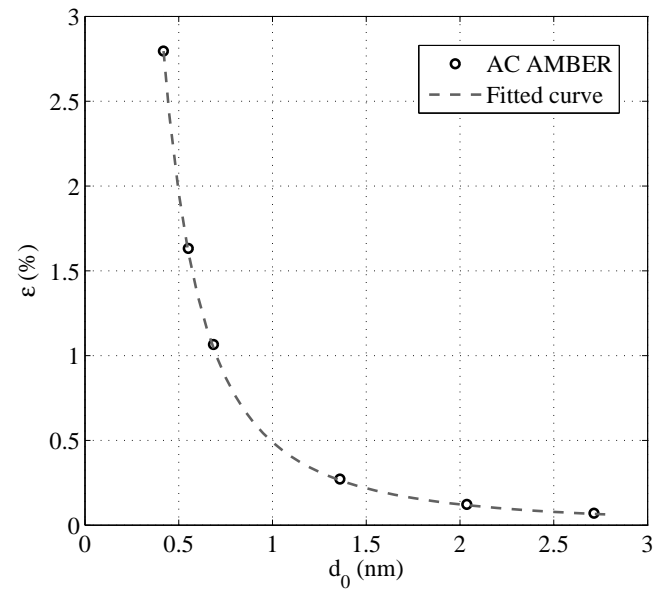

Fig. 6 Relative error against diameter (Armchair, AMBER)

Applying the minimum condition (7) to (31) and using (28), we achieve:

$$
\begin{aligned}
\Delta \theta_{1}\left[1+3 k_{s}\left(\Delta \theta_{1}\right)^{4}\right] & \frac{C_{2 m} \cos \alpha}{\sqrt{1-\left(C_{2 m} \sin \alpha\right)^{2}}}- \\
& -\Delta \theta_{2}\left[1+3 k_{s}\left(\Delta \theta_{2}\right)^{4}\right]=0,
\end{aligned}
$$

where $\Delta \theta_{1}, \Delta \theta_{2}$ are related to $\alpha$ through (28). Therefore, $\alpha$ is the unique unknown in equation (32), which has been solved numerically for $a_{0}=0.142 \mathrm{~nm}$. Substituting the obtained value of $\alpha$ in (24), the diameter $d_{0}$ is obtained. As in the case for Zig-zag nanotubes, the values of $d_{0}$ with Morse potential are the same as those in table 2 due to the same cause (see appendix B, table 7). Therefore, it is shown that Morse potential does not introduce any difference in the obtention of the initial diameter for Armchair SWCNTs.

\section{Initial diameter for Chiral nanotubes}

In this section, the obtention of the initial diameter $d_{0}$ for Chiral nanotubes (referred to as $\mathrm{CH}(\mathrm{n}, \mathrm{m})$ ) is tackled from a different point of view. It is worth noting that $\mathrm{CH}$ nanotubes do not present the property of axisymmetry (contrary to $\mathrm{ZZ}$ and $\mathrm{AC}$ nanotubes) and the procedure becomes noticeably more complicated. On the basis of this observation, the unstressed geometry of Chiral SWCNTs from the planar graphene sheet is conceptualized in two main steps: Firstly, a fictitious deformation pattern equivalent to an increment in diameter is imposed on the planar graphene sheet. Secondly, an aproximate mapping is applied to the deformed grid to configure the geometry of a Chiral nanotube, on which the minimization prodecure will be applied.

The parameters used in the calculations for $\mathrm{CH}$ nanotubes are defined in figures 7 and 8 .

The main assumptions related to the deformation procedure of the hexagonal grid are:

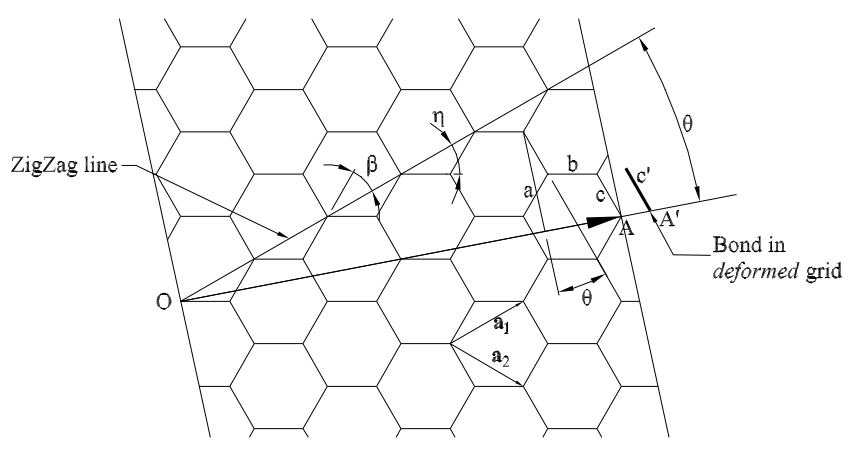

Fig. 7 Bond directions for the elemental system in $\mathrm{CH}$ nanotubes

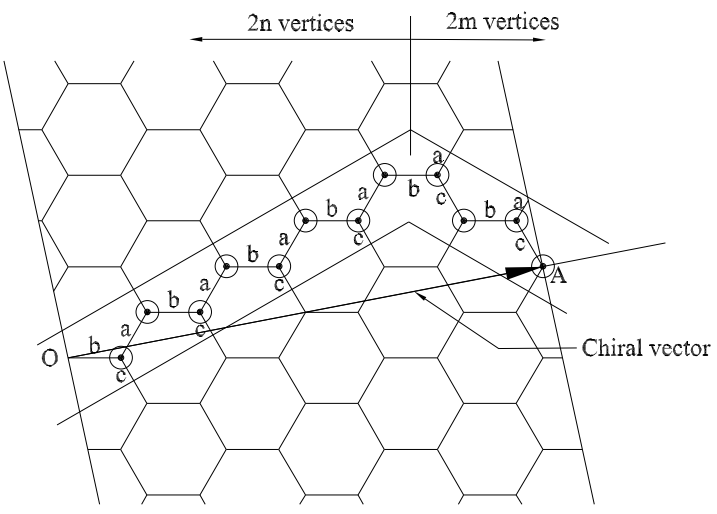

Fig. 8 Bond labels for the elemental system in $\mathrm{CH}$ nanotubes

1. The deformation pattern corresponds to a stretching of the grid in the circumferential direction OA of the nanotube.

2. Bonds labeled as c (orthogonal to the ZigZag line) keep their direction in the deformation process.

3. Angle $\beta$ has been chosen as independent parameter in the energy minimization.

4. All bonds keep their length in the deformation process (as assumed for $\mathrm{ZZ}$ and $\mathrm{AC}$ nanotubes).

5. The energy minimization is developed over the elemental strip represented in figure 8 , which can generate the whole geometry by periodicity in the longitudinal direction of the SWCNT.

Regarding assumptions 1, 2 and 4, we represent in figure 9 the deformation of an hexagonal unit cell produced by an imposed displacement $u$ in the OA direction.

In order to relate angle $\beta$ to $\eta$, the next compatibility equations in $\mathrm{P}$ are established:

$a_{0} \cos \beta+a_{0} \cos \eta=2 a_{0} \frac{\sqrt{3}}{2}+\delta_{x}$,

$a_{0} \sin \beta-a_{0} \sin \eta=-\delta_{y}$. 


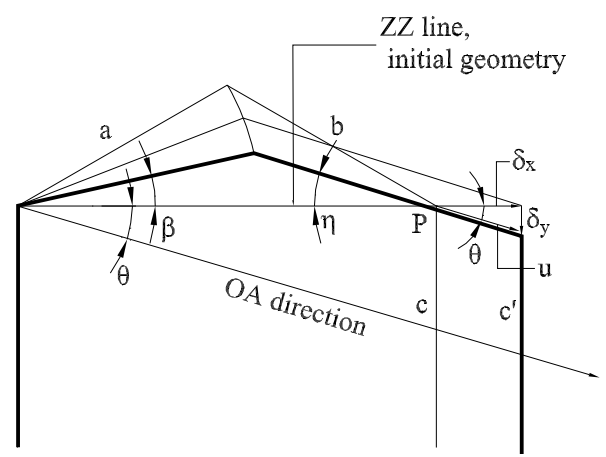

Fig. 9 Deformation pattern of an hexagonal unit cell

Dividing eqs.(33) by $a_{0}$ and writing $\delta_{x}, \delta_{y}$ in terms of the displacement $u$ :

$\cos \beta+\cos \eta=\sqrt{3}+\frac{u \cos \theta}{a_{0}}$,

$\sin \beta-\sin \eta=-\frac{u \sin \theta}{a_{0}}$

Removing the parameter $u$ from (34)

$\sin (\theta+\beta)+\sin (\theta-\eta)=\sqrt{3} \sin \theta$

which yields

$\eta=\theta-\arcsin [\sqrt{3} \sin \theta-\sin (\theta+\beta)]$.

Furthermore, angles comprised between neighboring bonds should be related to the angle $\beta$ (assumption 3). For this purpose, the diagrams depicted in figure 10 and 11 are used. Aimed to convert the planar distorted grid into the nanotube geometry, an approximate mapping that keeps the relative orientations of bonds with respect to the axial and secant ${ }^{2}$ direction of the cylinder has been assumed.

Projecting the bonds in figure 10 onto the cross section (figure 11), the next relations should be verified:

$d_{a}=a_{0} \cos (\theta+\beta)$

$d_{b}=a_{0} \cos (\eta-\theta)$,

$d_{c}=a_{0} \sin \theta$.

Moreover, applying the theorem of sine over each triangle in figure 11

$d_{i}=d_{0} \sin \left(\frac{\varphi_{i}}{2}\right), \quad i=a, b, c$.

\footnotetext{
2 It results from the projection of the bond $\mathrm{AB}$ (or $\mathrm{BP}$ ) on the cross section of the cylinder that involves the nanotube
}

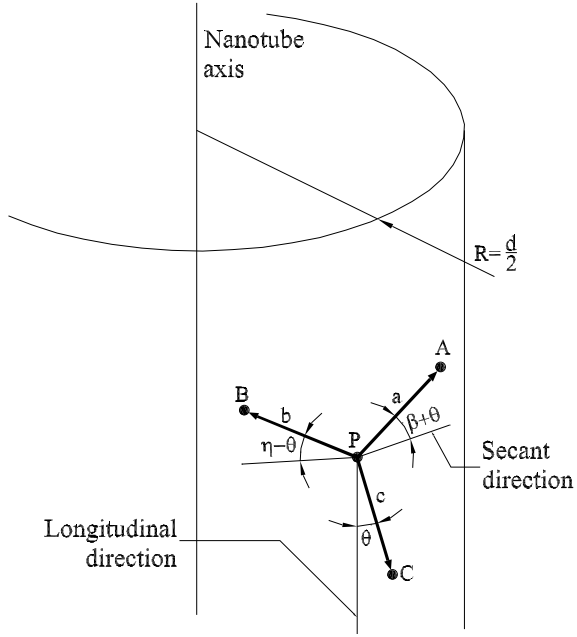

Fig. 10 Vectorial diagram of bonds $\mathrm{CH}$, perspective

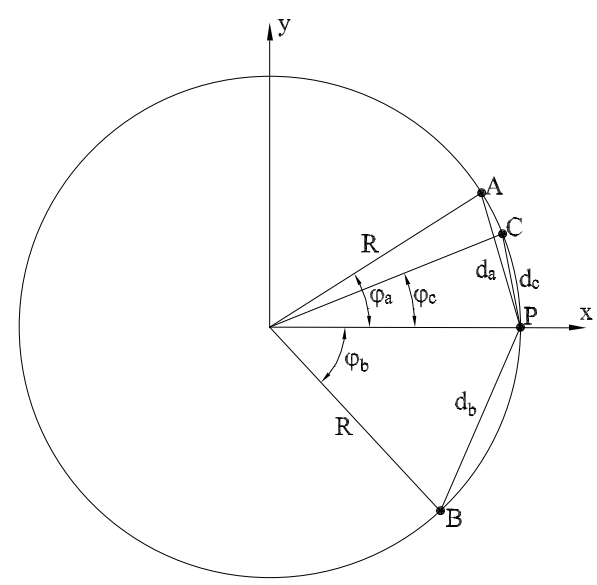

Fig. 11 Geometry diagram of bonds $\mathrm{CH}$, cross section

We introduce now the following auxiliary vectors between atoms:

$$
\mathbf{Z}_{1}=\overrightarrow{P A}=\left\{-d_{a} \sin \left(\frac{\varphi_{a}}{2}\right), d_{a} \cos \left(\frac{\varphi_{a}}{2}\right), a_{0} \sin (\theta+\beta)\right\}^{T}
$$

$$
\begin{aligned}
& \mathbf{Z}_{2}=\overrightarrow{P B}=\left\{-d_{b} \sin \left(\frac{\varphi_{b}}{2}\right),-d_{b} \cos \left(\frac{\varphi_{b}}{2}\right), a_{0} \sin (\eta-\theta)\right\}^{T} \\
& \mathbf{Z}_{3}=\overrightarrow{P C}=\left\{-d_{c} \sin \left(\frac{\varphi_{c}}{2}\right), d_{c} \cos \left(\frac{\varphi_{c}}{2}\right),-a_{0} \sin \theta\right\}^{T},
\end{aligned}
$$


whose dot products let us find out the required angles:

$$
\begin{aligned}
& \cos \theta_{a b}=\left(\frac{a_{0}}{d_{0}}\right)^{2} \cos ^{2}(\theta+\beta) \cos ^{2}(\eta-\theta)- \\
& -\cos (\theta+\beta) \cos (\eta-\theta) \sqrt{1-\left(\frac{a_{0}}{d_{0}}\right)^{2} \cos ^{2}(\theta+\beta)} . \\
& \cdot \sqrt{1-\left(\frac{a_{0}}{d_{0}}\right)^{2} \cos ^{2}(\eta-\theta)}+\sin (\theta+\beta) \sin (\eta-\theta),
\end{aligned}
$$

$$
\begin{aligned}
& \cos \theta_{a c}=\left(\frac{a_{0}}{d_{0}}\right)^{2} \sin ^{2} \theta \cos ^{2}(\theta+\beta)+ \\
& +\sin \theta \cos (\theta+\beta) \sqrt{1-\left(\frac{a_{0}}{d_{0}}\right)^{2} \sin ^{2} \theta} \\
& \cdot \sqrt{1-\left(\frac{a_{0}}{d_{0}}\right)^{2} \cos ^{2}(\theta+\beta)}-\cos \theta \sin (\theta+\beta),
\end{aligned}
$$

$$
\begin{aligned}
& \cos \theta_{b c}=\left(\frac{a_{0}}{d_{0}}\right)^{2} \sin ^{2} \theta \cos ^{2}(\eta-\theta)- \\
& -\sin \theta \cos (\eta-\theta) \sqrt{1-\left(\frac{a_{0}}{d_{0}}\right)^{2} \sin ^{2} \theta} . \\
& \cdot \sqrt{1-\left(\frac{a_{0}}{d_{0}}\right)^{2} \cos ^{2}(\eta-\theta)}-\cos \theta \sin (\eta-\theta) .
\end{aligned}
$$

Regarding the relation (36), expressions (40) to (42) can be written as functions of $\beta$. Therefore, the angular variations from the graphene sheet (involved in the interatomic potential) adopt the form:

$$
\begin{aligned}
\Delta \theta_{a b} & =\arccos \theta_{a b}(\beta)-\frac{2 \pi}{3}, \\
\Delta \theta_{a c} & =\arccos \theta_{a c}(\beta)-\frac{2 \pi}{3}, \\
\Delta \theta_{b c} & =\arccos \theta_{b c}(\beta)-\frac{2 \pi}{3} .
\end{aligned}
$$

In addition, the expression of the diameter $d_{0}$ written as a function of the angle $\beta$ is needed. From fig. 8, if we project the bonds included in the elemental strip over the OA direction, it yields for a $\mathrm{CH}(\mathrm{n}, \mathrm{m})$ :

$$
\begin{array}{ll}
\mathrm{n} & \text { segments of length } d_{b}, \\
\mathrm{n} & \text { segments of length } d_{a}, \\
\mathrm{~m} & \text { segments of length } d_{b}, \\
\mathrm{~m} & \text { segments of length } d_{c} .
\end{array}
$$

Thus, for the whole circumference can be established:

$$
\begin{gathered}
n \varphi_{a}+(n+m) \varphi_{b}+m \varphi_{c}=2 \pi, \\
n \frac{\varphi_{a}}{2}+m \frac{\varphi_{c}}{2}=\pi-(n+m) \frac{\varphi_{b}}{2} .
\end{gathered}
$$

Taking cosines in (44) and rearranging terms

$\cos \frac{n \varphi_{a}}{2} \cos \frac{m \varphi_{c}}{2}+\cos (n+m) \frac{\varphi_{b}}{2}=\sin \frac{n \varphi_{a}}{2} \sin \frac{m \varphi_{c}}{2}$,

relation that can be expressed in terms of the Chebyshev polynomials of the first kind as:

$$
\begin{aligned}
T_{n}\left(\cos \frac{\varphi_{a}}{2}\right) & T_{m}\left(\cos \frac{\varphi_{c}}{2}\right)+T_{n+m}\left(\cos \frac{\varphi_{b}}{2}\right)= \\
& =\frac{T_{n}^{\prime}\left(\cos \frac{\varphi_{a}}{2}\right) T_{m}^{\prime}\left(\cos \frac{\varphi_{c}}{2}\right)}{n m} \sin \frac{\varphi_{a}}{2} \sin \frac{\varphi_{c}}{2} .
\end{aligned}
$$

It should be noted that equation (46) connects the angles $\varphi_{i}$, which can be expressed as functions of the lengths $d_{i}$ through (38). These $d_{i}$, in turn, can be related to the orientations $\beta$ and $\eta$ by means of (37). Substituting then (36), there are only two parameters $\beta$ and $d_{0}$ left to be determined. Finally, the minimum condition corresponding to each potential provides the additional equation required to solve the problem.

\subsection{Energy minimization with AMBER potential}

Let $n_{v}$ be the total number of nodes (atoms) contained in the elemental strip represented in figure 8 , it is easy to show that the number of angles $\theta_{a b}, \theta_{a c}, \theta_{b c}$ of each kind included in the strip is the same and equal to $n_{v}=2 n+2 m$. Therefore, the expression ( 3 ) of the AMBER potential reduced to the elementary system is:

$U=\frac{n_{v}}{2} k_{\theta}\left(\Delta \theta_{a b}^{2}+\Delta \theta_{a c}^{2}+\Delta \theta_{b c}^{2}\right)$.

Applying equation (7) to (47) with respect to the parameter $\beta$ and introducing (43), the corresponding minimum condition can be expressed in terms of the unknowns $\beta$ and $d_{0}$. The aforementioned equation along with eq. (46) form a nonlinear system, which can be solved trough the iterative procedure in fig. 12

For the sake of completeness, the next remarks are convenient:

1. For the first step, the diameter $d_{0}^{0}=D_{t}$ defined from the conformal mapping and $\beta^{0}=\pi / 6$ corresponding to the planar (undistorted) graphene sheet are adopted as initial assumption.

2. The solution of each step is adopted as initial value for the Newton-like methods in the following iteration.

3. The maximum error adopted to accept the convergence is $\varepsilon=10^{-7}$ and for the Newton methods $\varepsilon=10^{-6}$.

The obtained results for different values of the integers $(\mathrm{n}, \mathrm{m})$ compared to those diameters $D_{t}$ from the conformal mapping, along with the relative error taking $d_{0}$ as exact solution, are outlined in table 3

As done in the previous cases, a curve proportional to $1 / R^{2}$ can be fitted to the relative errors, rendering $\varepsilon=0.1290 / R^{2}$. 


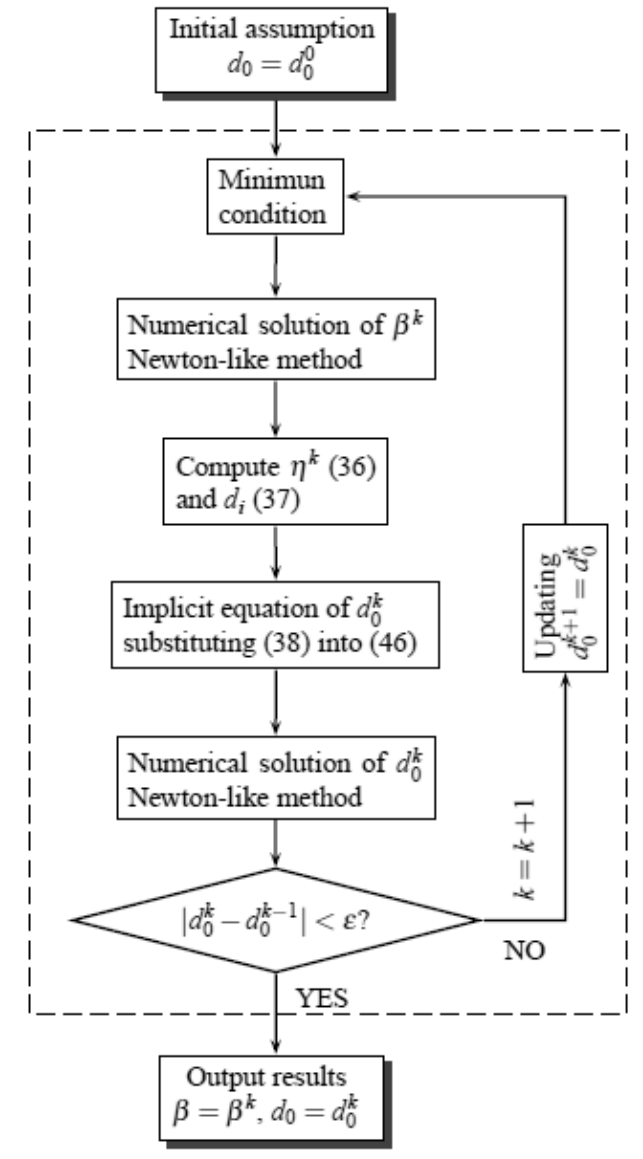

Fig. 12 Iterative procedure to solve $d_{0}$

\begin{tabular}{lcccccc}
\hline & $\mathrm{n}$ & $\mathrm{m}$ & $\beta(\mathrm{rad})$ & $d_{0}(\mathrm{~nm})$ & $D_{t}(\mathrm{~nm})$ & $\varepsilon(\%)$ \\
\hline $\mathrm{CH}(4,2)$ & 4 & 2 & 0.4864 & 0.4267 & 0.4143 & 2.9060 \\
$\mathrm{CH}(5,3)$ & 5 & 3 & 0.5037 & 0.5568 & 0.5480 & 1.5805 \\
$\mathrm{CH}(6,3)$ & 6 & 3 & 0.5061 & 0.6299 & 0.6214 & 1.3494 \\
$\mathrm{CH}(6,4)$ & 6 & 4 & 0.5116 & 0.6892 & 0.6825 & 0.9721 \\
$\mathrm{CH}(7,4)$ & 7 & 4 & 0.5125 & 0.7616 & 0.7550 & 0.8666 \\
$\mathrm{CH}(8,4)$ & 8 & 4 & 0.5136 & 0.8350 & 0.8285 & 0.7784 \\
\hline
\end{tabular}

Table 3 Initial diameters Chiral, AMBER. $D_{t}$ is the diameter associated to the conformal mapping, $d_{0}$ is the obtained diameter from the minimization procedure and $\varepsilon(\%)$ represents the relative error taking $d_{0}$ as the reference solution

For Chiral nanotubes, both curves are in reasonable agreement (figure 13), although some deviations are observed. This effect may be due to the lack of axisymmetry of $\mathrm{CH}$ nanotubes, which causes that the assumption of cylindrical geometry in absence of external loading is not as accurate as in ZigZag and Armchair cases. Moreover, the simplified mapping adopted in this section which keeps the relative orientations) introduces some error, mainly in nanotubes of small diameter.

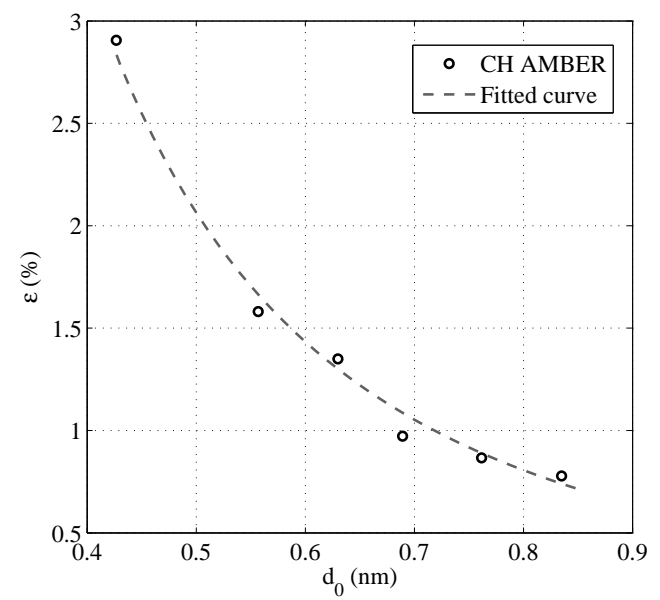

Fig. 13 Relative error against diameter (Chiral, AMBER)

\subsection{Energy minimization with Morse potential}

Reducing the expression (6) for the Morse potential to the elemental strip represented in figure 8 , it can be written:

$$
\begin{aligned}
& U=-\frac{3 n_{v}}{2} D_{e}+\frac{n_{v} k_{\theta}}{2}\left(\Delta \theta_{a b}\right)^{2}\left[1+k_{s}\left(\Delta \theta_{a b}\right)^{4}\right]+ \\
& +\frac{n_{v} k_{\theta}}{2}\left(\Delta \theta_{a c}\right)^{2}\left[1+k_{s}\left(\Delta \theta_{a c}\right)^{4}\right]+\frac{n_{v} k_{\theta}}{2}\left(\Delta \theta_{b c}\right)^{2}\left[1+k_{s}\left(\Delta \theta_{b c}\right)^{4}\right]
\end{aligned}
$$

Applying equation (7) to (48) with respect to $\beta$ and substituting (43), the parameters $\beta$ and $d_{0}$ remain to be solved in the minimum condition for Morse potential. Analogously to the AMBER case, the nonlinear system formed by such minimum condition and eq. (46) can be solved through a similar iterative procedure to that in figure 12. Also for $\mathrm{CH}$ nanotubes, the obtained values of $d_{0}$ from Morse potential are the same as those from AMBER in table 3 (see appendix B for further details). Therefore, the relative error represented in figure 13 and the conclusions for AMBER potential can be extended to this case.

\section{Concluding remarks}

In this paper, the geometry of single-walled carbon nanotubes in absence of any external load is studied via an energy approach. The initial geometry of nanotubes is assumed to be inscribed into a perfect cylinder of unknown diameter, which is determined by minimizing the interatomic potential. Two interatomic potential functions (AMBER and Morse) have been adopted for contrasting their influence in the final results. Our work is focused on developing a procedure able to estimate the more reasonable diameter, which can be decomposed in the following steps: firstly, the diameter of the nanotube is expressed as a function of several 
parameters describing the location of atoms into the cylindrical grid; secondly, these main parameters are reduced to one chosen as linearly independent; thirdly, the total energy contained in a representative system of the SWCNT is written as a function of the independent parameter; finally, the minimum condition of the total energy (eq. (7)) leads to the desired diameter.

Since the initial diameter is closely related to the preenergy and taking into account that strictly using the conformal mapping requires the introduction of a system of explicit internal forces (e.g. in MSM models as the stick-spiral, see $[15 \mid)$, the present method can be regarded as an alternative way to include the effect of these initial forces without evaluating them.

Finally, the main conclusions of this study are summarized next:

1. Our approach shows that the initial diameters of singlewalled nanotubes (with no external loading) are higher than those associated to the usually adopted conformal mapping (see e.g. $[5,7,14,28]$ ). This effect is more important in nanotubes with smaller diameters.

2. The obtained results show that the differences in nanotube diameter between the conformal mapping and those obtained from our minimization process are dependent on the diameter itself. The higher the nanotube diameter is, the lower difference is found. This conclusion is in agreement with other mechanically-justified size effects reported in the literature, as the increase in the Young modulus to an asymptotic value $|8,13-15|$, or the analogous decrease of the preenergy with increasing diameters $[8,20,23]$.

3 . The current minimization procedure yields a more reasonable approach to initial diameters of SWCNTs than other estimative calculations. This issue can be validated with theories of higher accuracy as tight-binding molecular dynamics. For instance, in table 1 of $|2|$ the diameter for a $\mathrm{ZZ}(15,0)$ is reported to be $1.2 \mathrm{~nm}$, closer to our value of $1.1802 \mathrm{~nm}$ (see table 1 ) than the value of 1.1743 $\mathrm{nm}$ from the conformal mapping. Likewise, in table 1 of $|8|$ a diameter of $0.791 \mathrm{~nm}$ is reported for a $\mathrm{ZZ}(10,0)$, closer to our value of $0.7925 \mathrm{~nm}$ than the value of 0.7829 $\mathrm{nm}$ from the conformal mapping.

4. The relative error from the conformal mapping to our calculations is proportional to $1 / R^{2}$, as has been shown in the paper for each chirality. This difference is related to the stabilization effect of the preenergy, which tends to transversally expand the nanotube. The smaller the diameter is, the higher effect of the preenergy is found, in agreement with $[8,20,23]$.

5. The adoption of AMBER or Morse potentials is almost inconsequential in the initial diameters, due to both functions having similar rigidities in the closeness of the equi- librium length of covalent bonds (accepted in the planar graphene sheet).

6. Although the fitting functions (they are of the form $\varepsilon=$ $k / R^{2}$ ) for the relative error are not identical for each chirality, they yield values of $k$ with the same order of magnitude. Therefore, the chirality does not have a remarkable influence on the initial diameters.

7. The comparison of obtained values in section A is useful for validating the assumptions made in the approximate mapping adopted for Chiral nanotubes. By taking the Chiral formulation to the ZigZag and Armchair cases, it is shown that (within the present minimization procedure) there is no significant influence of the particular mapping over the final results. The detailed study of the most physically reasonable mapping function has been deferred for further research.

\section{Appendix A Remarks on the coherence of the formulation}

In order to validate the assumptions adopted in the formulation for Chiral nanotubes, we compare the expressions and obtained results for Chiral SWCNTs (section 5) with those for ZigZag (section 3 ) and Armchair (section 4) nanotubes.

\section{A.1 Comparison Chiral-ZigZag}

$\mathrm{ZZ}(\mathrm{n}, 0)$ nanotubes are defined by an orientation of the Chiral vector $\theta=0$ and for the second integer $m=0$ in the base of the hexagonal grid. If $\theta$ vanishes in (35), it renders:

$\sin \eta=\sin \beta$

$\eta=\beta$.

Introducing (49) in equations (40) to (42) and operating:

$$
\begin{aligned}
& \cos \theta_{a b}=2\left(\frac{a_{0}}{d_{0}}\right)^{2} \cos ^{4} \beta-\cos ^{2} \beta+\sin ^{2} \beta, \\
& \cos \theta_{a c}=-\sin \beta \\
& \cos \theta_{b c}=-\sin \eta .
\end{aligned}
$$

As expected, it results $\theta_{a c}=\theta_{b c}=\theta_{1}$ in a coherent way with figure 1 . Besides, regarding definitions of $\alpha, \beta$ and $\eta$, it is obvious that $\alpha=\beta=\eta$. Therefore, from (9), it follows:

$\frac{a_{0}}{d_{0}} \cos \beta=\sin \left(\frac{\pi}{2 n}\right)$.

Substituting (51) into (50a) and operating:

$\cos \theta_{a b}=\sin ^{2} \beta\left(1+\cos \frac{\pi}{n}\right)-\cos \frac{\pi}{n}$.

The latter expression is completely equivalent to (12), therefore $\theta_{a b}=\theta_{2}$. 
Aimed to relate the diameter to $\beta$, we state $d_{a}=d_{b}$ and $d_{c}=0$ in (38), namely

$\varphi_{a}=\varphi_{b}, \quad \varphi_{c}=0$,

along with $m=0$, reduce (44) to:

$\frac{\varphi_{a}}{2}=\frac{\pi}{2 n}$

Taking sines in (54) and using (51):

$\sin \frac{\varphi_{a}}{2}=\sin \left(\frac{\pi}{2 n}\right)=\frac{a_{0}}{d_{0}} \cos \beta$,

achieving

$d_{0}=\frac{a_{0} \cos \beta}{\sin \left(\frac{\pi}{2 n}\right)}$

formally identical to (9).

Consequently, it has been shown that the general formulation for Chiral nanotubes reduced to the case ZigZag is equivalent to that developed in section 3 . Furthermore, some values from each formulation and the relative error between them (taking the direct formulation for $\mathrm{ZZ}$ nanotubes as a reference) are outlined in table 4

\begin{tabular}{lcccc}
\hline & & ZZ AMBER & CH AMBER & \\
& $\mathrm{n}$ & $d_{0}(\mathrm{~nm})$ & $d_{0}(\mathrm{~nm})$ & $\varepsilon_{d}(\%)$ \\
\hline $\mathrm{ZZ}(4,0)$ & 4 & 0.3356 & 0.3345 & 0.3504 \\
$\mathrm{ZZ}(5,0)$ & 5 & 0.4100 & 0.4095 & 0.1332 \\
$\mathrm{ZZ}(6,0)$ & 6 & 0.4854 & 0.4851 & 0.0610 \\
$\mathrm{ZZ}(10,0)$ & 10 & 0.7925 & 0.7924 & 0.0072 \\
$\mathrm{ZZ}(15,0)$ & 15 & 1.1807 & 1.1807 & 0.0017 \\
$\mathrm{ZZ}(20,0)$ & 20 & 1.5706 & 1.5706 & 0.0000 \\
\hline
\end{tabular}

Table 4 Comparison of obtained diameters Chiral-ZigZag, AMBER. $\varepsilon(\%)$ is the relative error taking the direct formulation for $\mathrm{ZZ}$ nanotubes as a reference

Taking into account that Morse potential does not introduce any difference in the obtained diameters, the corresponding results has not been included here.

The relative error can be attributed to: the accuracy of the iterative procedure (and in the Newton methods), the simplification of the mapping and the assumption of initial cylindrical geometry for Chiral nanotubes. However, from table 4 a good agreement between the two approaches has been shown.

\section{A.2 Comparison Chiral-Armchair}

In order to facilitate the comparison, the parameters defined on the hexagonal grid for both formulations (Chiral and Armchair) are represented together in figure 14

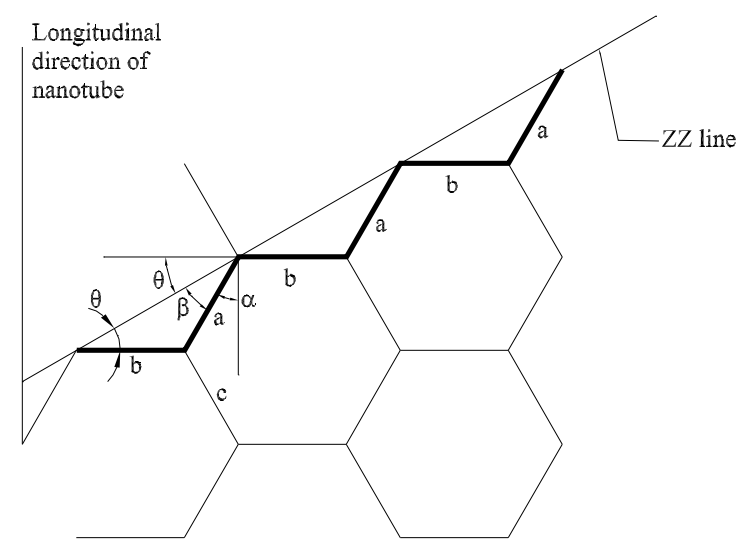

Fig. 14 Parameters definition, Chiral and Armchair formulations

An Armchair nanotube is defined by $n=m$, verifying the next angular relations:

$\eta=\theta$

$\beta+\theta=\frac{\pi}{2}-\alpha$,

which substituted in (35) lead to:

$\cos \alpha=\sqrt{3} \sin \theta$

In fact, (37) converts into:

$d_{a}=a_{0} \sin \alpha$

$d_{b}=a_{0}$,

$d_{c}=\frac{a_{0} \cos \alpha}{\sqrt{3}}$.

It is easy to show that figure 11 transformed into the cross section of an AC nanotube, would verify $d_{a}=d_{c}$. Nevertheless, from the Chiral formulation this condition is not verified in general, unless $\alpha=\pi / 6$ in (59). This value contradicts the target and the results of our formulation.

Consequently, both formulations are not identical regarding the final orientation of bonds into the distorted and mapped grid on the cylinder. In particular, the AC formulation keeps the orientation of bonds $\mathrm{b}$ (transversal direction) and $\mathrm{CH}$ formulation keeps the orientation of bonds c orthogonal to the $\mathrm{ZZ}$ line. If the deformation approached for $\mathrm{CH}$ nanotubes is converted to the AC case, two consecutive bonds b (perpendicular to the nanotube axis) are shifted a certain value along the axial direction of the nanotube.

Despite of that, projecting bonds $\mathrm{a}$ and $\mathrm{b}$ (thick line in figure 14) on the transversal direction of the nanotube up to complete the circumference, we can write:

$2 n \varphi_{a}+2 n \varphi_{b}=2 \pi$ 
equivalent to the relation obtained by imposing $n=m$ and $\varphi_{a}=\varphi_{c}$ (axisymmetry) in (44).

Dividing (60) by $4 n$ and taking trigonometric functions:

$\sin \left(\frac{\varphi_{a}}{2}+\frac{\varphi_{b}}{2}\right)=\sin \frac{\varphi_{a}}{2} \cos \frac{\varphi_{b}}{2}+\sin \frac{\varphi_{b}}{2} \cos \frac{\varphi_{a}}{2}=\sin \frac{\pi}{2 n}$

$\cos \left(\frac{\varphi_{a}}{2}+\frac{\varphi_{b}}{2}\right)=\cos \frac{\varphi_{a}}{2} \cos \frac{\varphi_{b}}{2}-\sin \frac{\varphi_{b}}{2} \sin \frac{\varphi_{a}}{2}=\cos \frac{\pi}{2 n}$.

Squaring (61) and substituting (62) leads to:

$$
\begin{aligned}
\sin ^{2} \frac{\varphi_{a}}{2} \cos ^{2} \frac{\varphi_{b}}{2}+ & \cos ^{2} \frac{\varphi_{a}}{2} \sin ^{2} \frac{\varphi_{b}}{2}+2 \sin ^{2} \frac{\varphi_{a}}{2} \sin ^{2} \frac{\varphi_{b}}{2}+ \\
& +2 \sin \frac{\varphi_{a}}{2} \sin \frac{\varphi_{b}}{2} \cos \frac{\pi}{2 n}=\sin ^{2} \frac{\pi}{2 n}
\end{aligned}
$$

Introducing now (59a) and (59b) in (38), we reach:

$\sin \frac{\varphi_{a}}{2}=\frac{a_{0} \sin \alpha}{d_{0}}$

$\sin \frac{\varphi_{b}}{2}=\frac{a_{0}}{d_{0}}$.

Using (64) in (63) along with (25) and operating, it can be obtained:

$d_{0}=\frac{a_{0}}{S_{2 n}} \sqrt{1+\sin ^{2} \alpha+2 \sin \alpha C_{2 n}}$,

identical to (24). Therefore, in spite of the aforementioned differences regarding the orientation of bonds, the obtained diameter must be the same from both procedures. For checking this issue, several diameters with AMBER potential by using each formulation and the relative error taking the direct formulation of $\mathrm{AC}$ nanotubes as a reference, are included in table 5

\begin{tabular}{lcccc}
\hline & & AC AMBER & CH AMBER & \\
& $\mathrm{n}=\mathrm{m}$ & $d_{0}(\mathrm{~nm})$ & $d_{0}(\mathrm{~nm})$ & $\varepsilon_{d}(\%)$ \\
\hline $\mathrm{AC}(3,3)$ & 3 & 0.4185 & 0.4154 & 0.7407 \\
$\mathrm{AC}(4,4)$ & 4 & 0.5514 & 0.5489 & 0.4534 \\
$\mathrm{AC}(5,5)$ & 5 & 0.6853 & 0.6833 & 0.2918 \\
$\mathrm{AC}(10,10)$ & 10 & 1.3597 & 1.3586 & 0.0809 \\
$\mathrm{AC}(15,15)$ & 15 & 2.0365 & 2.0358 & 0.0344 \\
\hline
\end{tabular}

Table 5 Comparison of obtained diameters Chiral-Armchair, AMBER. $\varepsilon(\%)$ is the relative error taking the direct formulation for AC nanotubes as a reference

Since Morse potential produces nearly the same values of diameters, only results for AMBER potential has been included here.

The relative error can be associated to the accuracy of the numerical process and the adopted mapping for Chiral nanotubes, similarly to the comparison Chiral-ZigZag. Also in this case, a good agreement has been shown from table 5 .
As has been proved in this section, different mappings and distortions of the hexagonal grid can produce nearly the same diameters in absence of external loading. Therefore, both specific deformation pattern and mapping can be assumed without loss of generality. The study of the physically more reasonable mapping has been deferred for further research.

\section{Appendix B Error estimation between potentials into the minimum condition}

Aimed to clarify the independency of our formulation on the adopted interatomic potential, an error estimation is worked out on the basis of Taylor expansion for both potentials. Thus, the Taylor expansion of order two for AMBER potential $\left(U_{a}\right)$ is the own eq. (3) and the analogous expansion for Morse potential can be written from eq. (6) as:

$U_{m}=U_{m, 0}+\sum_{j} \frac{1}{2} k_{\theta}\left(\Delta \theta_{j}\right)^{2}+\sum_{j} E_{m j, 2}$,

where

$$
\begin{array}{ll}
U_{m, 0}=-\sum_{i} D_{e} & \text { Constant term } \\
E_{m j, 2}=\frac{1}{2} k_{\theta} k_{s}\left(\Delta \theta_{j}\right)^{6} & \begin{array}{l}
\text { Remainder of the Taylor } \\
\text { expansion. }
\end{array}
\end{array}
$$

Applying the minimum condition (7) to AMBER potential with respect to a generic independent parameter $\xi$ and introducing a functional $\phi_{a}\left(U_{a}, \xi\right)$, it yields:

$\phi_{a}=\sum_{j} \Delta \theta_{j} \frac{\partial\left(\Delta \theta_{j}\right)}{\partial \xi}=0$.

As shown, the minimum condition does not depend on $k_{\theta}$. Likewise, applying condition (7) to (66) for Morse potential and defining a new functional $\phi_{m}\left(U_{m}, \xi\right)$, we reach:

$\phi_{m}=\sum_{j} \Delta \theta_{j} \frac{\partial\left(\Delta \theta_{j}\right)}{\partial \xi}+\sum_{j} 3 k_{s}\left(\Delta \theta_{j}\right)^{5} \frac{\partial\left(\Delta \theta_{j}\right)}{\partial \xi}=0$.

Subtracting (67) from (68), the absolute error in the minimum condition between both potentials can be defined by:

$\varepsilon_{\phi}=\left|\phi_{m}-\phi_{a}\right|=3 k_{s}\left|\sum_{j}\left(\Delta \theta_{j}\right)^{5} \frac{\partial\left(\Delta \theta_{j}\right)}{\partial \xi}\right|$.

For Zig-zag nanotubes, the angle $\alpha$ (see fig. 1) was taken as independent parameter, namely $\xi=\alpha$. Hence, evaluating $\Delta \theta_{j}$ and its partial derivatives from eqs. (13), the next values of the absolute error $\varepsilon_{\phi}$ are obtained:

For Armchair nanotubes, the angle $\alpha$ defined in fig. 4 was chosen as independent parameter. Therefore, computing $\Delta \theta_{j}$ and its partial derivatives from eqs. (28), the absolute error $\varepsilon_{\phi}$ renders: 


\begin{tabular}{lccc}
\hline & $\mathrm{m}$ & $\alpha(\mathrm{rad})$ & $\varepsilon_{\phi}(\mathrm{nN} \cdot \mathrm{nm} / \mathrm{rad})$ \\
\hline $\mathrm{ZZ}(4,0)$ & 4 & 0.4406 & $3.965 \cdot 10^{-4}$ \\
$\mathrm{ZZ}(5,0)$ & 5 & 0.4685 & $2.988 \cdot 10^{-5}$ \\
$\mathrm{ZZ}(6,0)$ & 6 & 0.4848 & $3.679 \cdot 10^{-6}$ \\
$\mathrm{ZZ}(10,0)$ & 10 & 0.5094 & $1.148 \cdot 10^{-8}$ \\
$\mathrm{ZZ}(15,0)$ & 15 & 0.5173 & $1.255 \cdot 10^{-10}$ \\
$\mathrm{ZZ}(20,0)$ & 20 & 0.5200 & $5.118 \cdot 10^{-12}$ \\
\hline
\end{tabular}

Table 6 Absolute error in the minimum condition for $\mathrm{ZZ}$ nanotubes

\begin{tabular}{lccc}
\hline & $\mathrm{m}$ & $\alpha(\mathrm{rad})$ & $\varepsilon_{\phi}(\mathrm{nN} \cdot \mathrm{nm} / \mathrm{rad})$ \\
\hline $\mathrm{AC}(3,3)$ & 3 & 0.5470 & $1.355 \cdot 10^{-5}$ \\
$\mathrm{AC}(4,4)$ & 4 & 0.5375 & $6.263 \cdot 10^{-7}$ \\
$\mathrm{AC}(5,5)$ & 5 & 0.5327 & $5.573 \cdot 10^{-8}$ \\
$\mathrm{AC}(10,10)$ & 10 & 0.5260 & $2.848 \cdot 10^{-11}$ \\
$\mathrm{AC}(15,15)$ & 15 & 0.5246 & $3.692 \cdot 10^{-12}$ \\
$\mathrm{AC}(20,20)$ & 20 & 0.5242 & $1.304 \cdot 10^{-14}$ \\
\hline
\end{tabular}

Table 7 Absolute error in the minimum condition for AC nanotubes

\begin{tabular}{lcccc}
\hline & $\mathrm{n}$ & $\mathrm{m}$ & $\beta(\mathrm{rad})$ & $\varepsilon_{\phi}(\mathrm{nN} \cdot \mathrm{nm} / \mathrm{rad})$ \\
\hline $\mathrm{CH}(4,2)$ & 4 & 2 & 0.4864 & $6.965 \cdot 10^{-6}$ \\
$\mathrm{CH}(5,3)$ & 5 & 3 & 0.5037 & $6.239 \cdot 10^{-7}$ \\
$\mathrm{CH}(6,3)$ & 6 & 3 & 0.5061 & $7.380 \cdot 10^{-8}$ \\
$\mathrm{CH}(6,4)$ & 6 & 4 & 0.5116 & $1.850 \cdot 10^{-7}$ \\
$\mathrm{CH}(7,4)$ & 7 & 4 & 0.5125 & $4.422 \cdot 10^{-8}$ \\
$\mathrm{CH}(8,4)$ & 8 & 4 & 0.5136 & $1.152 \cdot 10^{-8}$ \\
\hline
\end{tabular}

Table 8 Absolute error in the minimum condition for $\mathrm{CH}$ nanotubes

Finally, the angle $\beta$ defined in fig. 7 was adopted as independent parameter for Chiral nanotubes and employing (43) in (69), the next values of $\varepsilon_{\phi}$ were calculated:

As shown in tables 6 to 8 , almost the whole values of the absolute error verify $\varepsilon_{\phi}<10^{-4} \mathrm{nN} \cdot \mathrm{nm} / \mathrm{rad}$ (except for $\mathrm{ZZ}(4,0))$, which was the accuracy adopted in the obtained diameters in this paper. Therefore, the obtained diameters with AMBER and Morse potentials were almost identical for each chirality, regarding four significant digits.

\section{References}

1. Arroyo M, Belytschko T (2003) Nonlinear mechanical response and rippling of thick multiwalled carbon nanotubes. Phys. Rev. Lett. 91:215505-215508.

2. Bagolini L, Gala F, Zollo G (2012) Methane cracking on singlewall carbon nanotubes studied by semi-empirical tigh binding simulations. Carbon 50:411-420.

3. Belytschko T, Xiao SP, Schatz GC et al (2002) Atomistic simulations of nanotube fracture. Phys. Rev. B 65:235430-235437

4. Benvenuti E (2015) Elechtromechanical behavior, end enhancements and radial elasticity of single-walled CNTs: A physicallyconsistent model based on nonlocal charges. Int. J. Solids Struct. 72:190-205

5. Chang T, Gao H (2003) Size-dependent elastic properties of a single-walled carbon nanotube via a molecular mechanics model. J. Mech. Phys. Solids 51:1059-1074

6. Chang T, Geng J, Guo X (2006) Prediction of chirality- and sizedependent elastic properties of single-walled carbon nanotubes via a molecular mechanics model. Proc. R. Soc. A 462:2523-2540
7. Dresselhaus MS, Dresselhaus G, Avouris P (2001) Carbon Nanotubes: Synthesis, Structure, Properties, and Applications. Topics in Applied Physics (vol 80), Springer, Berlin

8. Hernández E, Goze C, Bernier P et al (1998) Elastic properties of $\mathrm{C}$ and $B_{x} C_{y} N_{z}$ composite Nanotubes. Phys. Rev. Lett. 80:4502-4505

9. Iijima S, Brabec C, Maiti A et al (1996) Structural flexibility of carbon nanotubes. J. Chem. Phys. 104:2089-2092

10. Li C, Chou TW (2003) A structural mechanics approach for the analysis of carbon nanotubes. Int. J. Solids Struct. 40:2487-2499

11. Li C, Chou TW (2003) Elastic moduli of multi-walled carbon nanotubes and the effect of van der waals forces. Compos. Sci. Technol. 63:1517-1524

12. Li X, Yang W, Liu B (2007) Bending Induced Rippling and Twisting of Multiwalled Carbon Nanotubes. Phys. Rev. Lett. 98:205502-205505

13. Malagú M, Benvenuti E, Simone A (2015) One-dimensional nonlocal elasticity for tensile single-walled carbon nanotubes: A molecular structural mechanics characterizaton. Eur. J. Mech. A Solids. 54:160-170.

14. Meo M, Rossi M (2006) Prediction of young's modulus of single wall carbon nanotubes by molecular-mechanics based finite element modelling. Compos. Sci. Technol. 66:1597-1605

15. Merli R, Lázaro C, Monleón S et al (2013) A Molecular Structural Mechanics model applied to the static behavior of single-walled Carbon nanotubes: New general formulation. Comput. \& Struct. 127:68-87

16. Merli R, Lázaro C, Monleón S et al (2015) Geometrical nonlinear formulation of a Molecular Mechanics model applied to the structural analysis of single-walled carbon nanotubes. Int. J. Solids Struct. 58:157-177

17. Natsuki T, Tantrakarn K, Endo M (2004) Prediction of elastic properties for singlewalled carbon nanotubes. Carbon 42:39-45

18. Natsuki T, Endo M (2004) Stress simulation of carbon nanotubes in tension and compression. Carbon 42:2147-2151

19. Odegard GM, Gates TS, Nicholson LM et al (2002) Equivalentcontinuum modeling of nano-structured materials. Compos. Sci. Technol. 62:1869-1880

20. Pantano A, Parks DM, Boyce MC (2004) Mechanics of deformation of single- and multi-wall carbon nanotubes. J. Mech. Phys. Solids 52:789-821

21. Rochefort A, Avouris P, Lesage F et al (1999) Electrical and mechanical properties of distorted carbon nanotubes. Phys. Rev. B 60:13824-13830

22. Paulson S, Falvo MR, Snider N et al (1999) In situ resistance measurements of strained carbon nanotubes. Appl. Phys. Lett. 75:2936-2938

23. Robertson DH, Brenner DW, Mintmire JW (1992) Energetics of nanoscale graphitic tubules. Phys. Rev. B 45:12592-12595

24. Srivastava D, Menon M, Cho K (1999) Nanoplasticity of singlewall carbon nanotubes under uniaxial compression. Phys. Rev. Lett. 83:2973-2976

25. Tersoff J, Ruoff RS (1994) Structural properties of a carbonnanotube crystal. Phys. Rev. Lett. 73:676-679

26. Wang Q (2004) Effective in-plane stiffness and bending rigidity of armchair and zigzag carbon nanotubes. Int. J. Solids Struct. 41:5451-5461

27. Wang X, Wang X, Xiao J (2005) A non-linear analysis of the bending modulus of carbon nanotubes with rippling deformations. Compos. Struct. 69:315-321

28. Xiao J, Gama B, Gillespie Jr. J (2005) An analytical molecular structural mechanics model for the mechanical properties of carbon nanotubes. Int. J. Solids Struct. 42:3075-3092

29. Yakobson BI, Brabec CJ, Bernholc J (1996) Nanomechanics of carbon tubes: Instabilities beyond linear response. Phys. Rev. Lett. 76:2511-2514 
30. Zaeri MM, Ziaei-Rad S, Vahedi A et al (2010) Mechanical modelling of carbon nanomaterials from nanotubes to buckypaper. Carbon 48:3916-3930. 